\title{
POINT DERIVATIONS ON FUNCTION ALGEBRAS GENERATED BY HOLOMORPHIC FUNCTIONS
}

\author{
R. G. M. BRUMMELHUIS AND P. J. DE PAEPE
}

(Communicated by Paul S. Muhly)

\begin{abstract}
It is shown that a continuous point derivation on the algebra $H(X)$ consisting of uniform limits on $X$ of functions holomorphic in a neighborhood of a compact subset $X$ in $\mathbf{C}^{n}$, which vanishes on the polynomials is the trivial derivation.
\end{abstract}

\section{INTRODUCTION}

Let $X$ be a compact Hausdorff space. A function algebra $B$ on $X$ is a point separating sup norm closed subalgebra of $C(X)$ (the algebra of all complexvalued continuous functions on $X$ ) containing the constant functions on $X$. $\Delta B$ will denote the maximal ideal space of $B$, i.e. the space of nontrivial complex homomorphisms of $B$.

A continuous point derivation of $B$ at some element $\phi \in \Delta B$ is a continuous linear functional $D$ on $B$ such that

$$
D f g=\phi(f) D g+\phi(g) D f
$$

for all $f, g \in B$. The collection of all continuous point derivations of $B$ at $\phi$ is a linear subspace $\mathscr{D}(B, \phi)$ of $B^{*}$.

For a compact subset $X$ of $\mathbf{C}^{n}, P(X)$ will denote the uniform closure in $C(X)$ of the polynomials (considered as functions on $X$ ) and $H(X)$ will be the closure in $C(X)$ of the collection of functions holomorphic in some neighborhood of $X$.

Using results and ideas of [BdP] we will prove a result which shows that a continuous point derivation of $H(X)$ is completely determined by its values on the polynomials:

Theorem. Let $X$ be a compact subset of $\mathbf{C}^{n}$. Let $D$ be a continuous point derivation of $H(X)$ such that $D \mid P(X)=0$. Then $D=0$.

Received by the editors January $28,1988$.

1980 Mathematics Subject Classification (1985 Revision). Primary 32A10, 46J10.

The first author was supported by the Netherlands Organization for the Advancement of Pure Research (ZWO). 


\section{Proof of THE THEOREM}

Let $U$ be an open subset of $\mathbf{C}^{n}$, containing $X$. Let $E(U)$ be the envelope of holomorphy of $U$ (see [GR] for basic facts on the theory of several complex variables). Every element $f$ of the algebra $\mathscr{O}(U)$ of holomorphic functions on $U$ can be extended in a unique way to an element $\hat{f}$ of $\mathscr{O}(E(U))$. Now let $\phi \in \Delta H(X)$. The map $f \rightarrow \phi(f \mid X)$ from $\mathscr{O}(E(U))$ onto $\mathbf{C}$ defines a continuous homomorphism of $\mathscr{O}(E(U)$ ) (endowed with the topology of uniform convergence on compact subsets of $E(U))$, and since these are the point evaluations at the points of $E(U),[\mathrm{GR}$, Chapter $\mathrm{I}, \S \mathrm{G}]$, there is a point $a \in E(U)$ such that

$$
\phi(f \mid X)=f(a)
$$

for all $f \in \mathscr{O}(E(U))$. Now let $D \in \mathscr{D}(H(X), \phi)$ with $D \mid P(X)=0$. Defining $d: \mathscr{O}(E(U)) \rightarrow \mathbf{C}$ by $d f=D(f \mid X)$ it follows that $d$ is linear and satisfies

$$
d(f g)=f(a) d g+g(a) d f
$$

for all $f, g \in \mathscr{O}(E(U))$.

Moreover $d(\hat{p})=0$ for any polynomial $p$ on $\mathbf{C}^{n}$. We now argue as in [BdP, Proof of Theorem 1]. By [H, Theorem 5.3.9, p. 128] there exist $f_{1}, \ldots, f_{m} \in$ $\mathscr{O}(E(U))$ such that the map $\Phi=\left(\hat{z}_{1}, \ldots, \hat{z}_{n}, f_{1}, \ldots, f_{m}\right): E(U) \rightarrow \mathbf{C}^{n+m}$ is one-to-one and proper. Applying [GR, Theorem 15, Chapter VIII, §A, p. 224] to the ideal sheaf of $\{a\}$, we find that for any $f \in \mathscr{O}(E(U))$ there exist functions $h_{1}, \ldots, h_{n}, g_{1}, \ldots, g_{m} \in \mathscr{O}(E(U))$ such that

$$
f-f(a)=\sum_{i=1}^{n}\left(\hat{z}_{i}-\hat{z}_{i}(a)\right) h_{i}+\sum_{i=1}^{m}\left(f_{i}-f_{i}(a)\right) g_{i} .
$$

Applying $d$ to (1), using $d \hat{z}_{i}=0, i=1, \ldots, n$, we obtain

$$
d f=\sum_{i=1}^{m} g_{i}(a) d f_{i},
$$

i.e. for any $f \in \mathscr{O}(E(U)) d f$ is a linear combination of the numbers $d f_{1}, \ldots$, $d f_{m}$. Now let $k \in \mathbf{N}, H \in \mathscr{O}\left(\mathbf{C}^{k}\right), b \in \mathbf{C}^{k}$. Then there are functions $H_{1}, \ldots$, $H_{k} \in \mathscr{O}\left(\mathbf{C}^{k}\right)$ such that

$$
H(\zeta)=H(b)+\sum_{i=1}^{k}\left(\zeta_{i}-b_{i}\right) H_{i}(\zeta)
$$

again by the above cited theorem in [GR], or more simply in this case, using power series. Note: $H_{i}(b)=\frac{\partial H}{\partial \xi_{i}}(b), i=1, \ldots, n$. Now in (3) we take $k=n+m, \zeta=\Phi(z), b=\Phi(a)$ and $H \in \mathscr{O}\left(\mathbf{C}^{n+m}\right)$, and apply $d$.

It follows that with standard coordinates $\left(z_{1}, \ldots, z_{n}, w_{1}, \ldots, w_{m}\right) \in \mathbf{C}^{n} \times$ $\mathbf{C}^{m}=\mathbf{C}^{n+m}$,

$$
d H\left(\hat{z}_{1}, \ldots, \hat{z}_{n}, f_{1}, \ldots, f_{m}\right)=\sum_{i=1}^{m} \frac{\partial H}{\partial w_{i}}(\Phi(a)) d f_{i} .
$$


Applying Theorem A of Cartan [GR, Theorem 13, Chapter VIII, §A, p. 243] to the sheaf of ideals of $\widetilde{V}=\Phi(E(U))$, it can be shown that given $x \in \widetilde{V}$ there are $H_{1}, \ldots, H_{m} \in \mathscr{O}\left(\mathbf{C}^{n+m}\right)$ with $H_{i}$ vanishing on $\widetilde{V}(i=1, \ldots, m)$ such that $\left(\partial\left(H_{1}, \ldots, H_{m}\right) / \partial\left(w_{1}, \ldots, w_{m}\right)\right)(x) \neq 0$. In particular for the point $a \in E(U)$ we find $H_{1}, \ldots, H_{m} \in \mathscr{O}\left(\mathbf{C}^{n+m}\right)$, vanishing on $\tilde{V}$ such that

$$
\frac{\partial\left(H_{1}, \ldots, H_{m}\right)}{\partial\left(w_{1}, \ldots, w_{m}\right)}(\Phi(a)) \neq 0 .
$$

By (4) $\sum_{i=1}^{m} \frac{\partial H_{j}}{\partial u_{i}}(\boldsymbol{\Phi}(a)) d f_{i}=0, j=1, \ldots, m$.

From (5) it now follows that $d f_{i}=0, i=1, \ldots, m$, so by (2): $d f=0$ for all $f \in \mathscr{O}(E(U)$. So $D|\mathscr{O}(U)| X=0$, so by continuity $D=0$.

Remark. For the special case that $X$ is holomorphically convex, i.e. $\Delta H(X)=$ $X$, we can give a short function algebraic proof of the theorem: First, we recall a well-known fact: if $B$ is a function algebra on $X$ with $P(X) \subset B \subset H(X)$ and with $\Delta B=X$, then $B=H(X)$. Indeed: the functions $z_{1}, \ldots, z_{n}$ belong to $B$ and the joint spectrum $\sigma_{B}\left(z_{1}, \ldots, z_{n}\right)$ equals $X$ since $\Delta B=X$. So if $f \in \mathscr{O}(U), U$ open in $C^{n}$ and containing $X$, by the functional calculus [G2] $f \circ\left(\hat{z}_{1}, \ldots, \hat{z}_{n}\right) \in \widehat{B}$ (here $\hat{g}$ denotes the Gelfand transform of $g \in B$ ), so $f \in B$. Hence $\mathscr{O}(U) \mid X \subset B$, so $B=H(X)$. Now assume $\Delta H(X)=X$ and $D$ is a continuous point derivation on $H(X)$ with $D \mid P(X)=0$. Let $B=\operatorname{Ker} D$. The kernel of $D$ is a function algebra on $X$ containing $P(X)$ and $\Delta D=\Delta H(X)$, cf. [G1]. By the above remark: $\operatorname{Ker} D=H(X)$, i.e. $D=0$.

\section{EXAMPLeS}

Since the theorem shows that any $D \in \mathscr{D}(H(X), \phi)$ is completely determined by its values on $P(X)$, it follows that $D$ is completely determined by $D\left(z_{1}\right), \ldots, D\left(z_{n}\right)$. This shows

Corollary. Let $X$ be a compact subset of $\mathbf{C}^{n}$ and $\phi \in \Delta H(X)$. Then

$$
\operatorname{dim} \mathscr{D}(H(X), \phi) \leq n .
$$

We give some simple examples.

(i) If $X$ is a compact subset of $\mathbf{C}^{n}$ and $a$ belongs to the interior of $X$, then for any $c_{1}, \ldots, c_{n} \in \mathbf{C},\left.\sum_{i=1}^{n} c_{i} \frac{\partial}{\partial z_{i}}\right|_{a}$ belongs to $\mathscr{D}(H(X), a)$, i.e. for an interior point $a \in X, \operatorname{dim} \mathscr{D}(H(X), a)=n$.

(ii) Let $X=\{z \in \mathrm{C}:|z| \leq 1\}$, then $P(X)=H(X)$. The continuous point derivations $D$ at points $a$ with $|a|<1$ are by example (i) of the form $D f=$ $c f^{\prime}(a), c \in \mathbf{C}$. Let $a$ be a boundary point of $X$, say $a=1$. Consider the function $f=\sqrt{1-z} \in H(X)$. If $D \in \operatorname{Der}(H(X), 1), D z=-D(1-z)=$ $-D f^{2}=2 f(1) D f=0$. Hence $D=0$. 
(iii) In a similar way, it follows that for

$$
\begin{array}{ll}
X=\left\{\left(z_{1}, z_{2}\right) \in \mathbf{C}^{2}:\left|z_{1}\right| \leq 1,\left|z_{1}\right| \leq 1\right\}, \\
\operatorname{dim} \mathscr{D}\left(H(X),\left(a_{1}, a_{2}\right)\right)=2 & \text { if }\left|a_{1}\right|<1,\left|a_{2}\right|<1, \\
\operatorname{dim} \mathscr{D}\left(H(X),\left(a_{1}, a_{2}\right)\right)=1 & \text { if }\left|a_{1}\right|=1,\left|a_{2}\right|<1 \text { or }\left|a_{2}\right|=1,\left|a_{1}\right|<1, \\
\operatorname{dim} \mathscr{D}\left(H(X),\left(a_{1}, a_{2}\right)\right)=0 & \text { if }\left|a_{1}\right|=\left|a_{2}\right|=1 .
\end{array}
$$

(iv) Let $X=\left\{\left(z_{1}, z_{2}\right) \in \mathbf{C}^{2}: z_{1}^{3}=z_{2}^{2},\left|z_{1}\right| \leq 1\right\}$. Suppose $D$ is a continuous point derivation of $P(X)=H(X)$ at $\left(a_{1}, a_{2}\right) \in X$. Since $z_{1}^{3}=z_{2}^{2}$ on $X$ we have $3 a_{1}^{2} D z_{1}=2 a_{2} D z_{2}$ so if $\left(a_{1}, a_{2}\right) \neq(0,0), D z_{2}=\left(3 a_{1}^{2} / 2 a_{2}\right) D z_{1}$, so it follows that

$$
\operatorname{dim} \mathscr{D}\left(H(X),\left(a_{1}, a_{2}\right)\right)=1 \quad \text { if }\left(a_{1}, a_{2}\right) \in X, 0<\left|a_{1}\right|<1
$$

and

$$
\operatorname{dim} \mathscr{D}\left(H(X),\left(a_{1}, a_{2}\right)\right)=0 \quad \text { if }\left(a_{1}, a_{2}\right) \in X,\left|a_{1}\right|=1 .
$$

Finally, $\mathscr{D}(H(X),(0,0))$ has dimension 2: if $f \in H(X), g(z)=f\left(z^{2}, z^{3}\right)$, $|z| \leq 1$, then $D_{1} f=g^{\prime \prime}(0), D_{2} f=g^{\prime \prime \prime}(0)$ define two linearly independent elements of $\mathscr{D}(H(X),(0,0))$.

\section{REFERENCES}

[BdP] R. G. M. Brummelhuis and P. J. de Paepe, Derivations on algebras of holomorphic functions, Department of Math., Univ. of Amsterdam, Preprint Series 87-23, 1987.

[G1] Th. Gamelin, Embedding Riemann surfaces in maximal ideal spaces. J. Funct. Anal. 2 (1968), 123-146.

[G2] Th. Gamelin, Uniform algebras, Prentice-Hall, 1969.

[GR] R. C. Gunning and H. Rossi, Analytic functions of several complex variables, Prentice-Hall, 1965.

[H] L. Hörmander, An introduction to complex analysis in several variables, North-Holland, 1973.

Department of Mathematics, University of Amsterdam, Roetersstraat 15, 1018 WB AMSTERDAM, The NetherLaNDS 